\section{International Scientific Journal Theoretical \& Applied Science}

\author{
p-ISSN: 2308-4944 (print) e-ISSN: 2409-0085 (online) \\ Year: $2016 \quad$ Issue: 4 Volume: 36 \\ Published: $30.04 .2016 \quad \underline{\text { http://T-Science.org }}$
}

SECTION 2. Applied mathematics. Mathematical modeling.
Yunona Rinatovna Krakhmaleva candidate of technical Sciences, associate Professor of Mathematics, Taraz state University named after M.Kh. Dulati,

Kazakhstan yuna_kr@mail.ru

Andrey Titovets master 2-year degree in Mathematics Taraz state University named after M.Kh. Dulati, Kazakhstan

\title{
TO THE QUESTION OF REDUCTION TO A CANONICAL FORM DIFFERENTIAL EQUATIONS IN PRIVATE DERIVATIVES WITH VARIABLE COEFFICIENTS IN MAPLE
}

Abstract: The article discusses some issues of analysis of differential equations with variable coefficients.

Key words: Maple, analysis, type, equation.

Language: Russian

Citation: Krakhmaleva YR, Titovets A (2016) TO THE QUESTION OF REDUCTION TO A CANONICAL FORM DIFFERENTIAL EQUATIONS IN PRIVATE DERIVATIVES WITH VARIABLE COEFFICIENTS IN MAPLE. ISJ Theoretical \& Applied Science, 04 (36): 197-200.

Soi: http://s-o-i.org/1.1/TAS-04-36-34 Doi: crossef http://dx.doi.org/10.15863/TAS.2016.04.36.34

\section{К ВОПРОСУ О ПРИВЕДЕНИИ К КАНОНИЧЕСКОМУ ВИДУ ДИФФЕРЕНЦИАЛЬНЫХ УРАВНЕНИЙ В ЧАСТНЫХ ПРОИЗВОДНЫХ С ПЕРЕМЕННЫМИ КОЭФФИЦИЕНТАМИ В CРЕДЕ МАPLE}

Аннотация: В статье рассматриваются некоторые вопросы анализа дифференциальных уравнений в частных производных с переменными коэффициентами.

Ключевые слова: Maple, анализ, вид, уравнение.

При составлении математической программы приведения к каноническому виду дифференциального уравнения в частных производных с постоянными коэффициентами в среде Maple 17 используются стандартные средства программы, такие как mapde (_, canom), mapde (_, canop), которые дают возможность создать универсальный алгоритм. При приведении к каноническому виду дифференциального уравнения в частных производных с переменными коэффициентами их задействовать возможно только, когда исходные уравнения довольно «простые». И хотя для работы с дифференциальными уравнениями в частных производных имеется достаточно большой набор инструментов пакета, математическая программа приведения к каноническому виду дифференциального уравнения в частных производных с переменными коэффициентами представляет сложную задачу.

Рассмотрим линейное уравнение в частных производных с переменными коэффициентами:

$a_{11}(x, y) \frac{\partial u}{\partial x^{2}}+2 a_{12}(x, y) \frac{\partial u}{\partial x \partial y}+a_{22}(x, y) \frac{\partial u}{\partial y^{2}}+a_{1}(x, y) \frac{\partial u}{\partial x}+a_{2}(x, y) \frac{\partial u}{\partial y}+a_{0}(x, y) u+d=0$

где $u=u(x, y) \quad$ - неизвестна функция.

Как известно, при классификации типов уравнений в частных производных вычисляют определитель $\triangle A$ квадратичной формы: $a_{11}(x, y) \frac{\partial u}{\partial x^{2}}+2 a_{12}(x, y) \frac{\partial u}{\partial x \partial y}+a_{22}(x, y) \frac{\partial u}{\partial y^{2}}=0$

знак которого определяет тип рассматриваемого уравнения: если $\Delta A>0$, то уравнение эллиптического типа; если $\Delta A<0$ то уравнение 


\begin{tabular}{|c|c|c|c|c|c|c|}
\hline Impact Factor: & $\begin{array}{l}\text { ISRA (India) } \\
\text { ISI (Dubai, UAE } \\
\text { GIF (Australia) } \\
\text { JIF }\end{array}$ & $\begin{array}{l}=1.344 \\
=0.829 \\
=0.564 \\
=1.500\end{array}$ & $\begin{array}{l}\text { SIS (USA) } \\
\text { PИНЦ (Russia) } \\
\text { ESJI (KZ) } \\
\text { SJIF (Morocco) }\end{array}$ & $\begin{array}{l}=0.912 \\
=0.234 \\
=1.042 \\
=2.031\end{array}$ & $\begin{array}{l}\text { ICV (Poland) } \\
\text { PIF (India) } \\
\text { IBI (India) }\end{array}$ & $\begin{array}{l}=6.630 \\
=1.940 \\
=4.260\end{array}$ \\
\hline
\end{tabular}

гиперболического типа; если $\Delta A=0$, то уравнение параболического типа:

$$
\begin{aligned}
& a 11:=\ldots b:=\ldots \text { a } 22:={ }_{\_} ; a 1:=\ldots a 2:=\ldots a 0:=\ldots d:=\ldots a 12:=\frac{b}{2} \text {; } \\
& \text { PDE1 := a11.diff(u(x,y), x, x })+2 \cdot \operatorname{al2} \cdot \operatorname{diff}(u(x, y), x, y)+a 22 \\
& \cdot \operatorname{diff}(u(x, y), y, y)+a 1 \cdot \operatorname{diff}(u(x, y), x)+a 2 \cdot \operatorname{diff}(u(x, y), y)+a 0 \\
& \cdot u(x, y)+d=0 \text {; } \\
& d t:=a 12^{2}-a 11 \cdot a 22 \\
& \text { if }(d t>0) \text { then uravnenie giperbolicheskogo tipa; fi; } \\
& \text { if }(d t<0) \text { then uravnenie elipticheskogo tipa; fi; } \\
& \text { if }(d t=0) \text { then uravnenie parabolicheskogo tipa; fi; }
\end{aligned}
$$

При составлении программ приведения дифференциального уравнения в частных производных с переменными коэффициентами к каноническому виду средствами Maple многие разработчики предлагают не использовать выше написанную классификацию, а определять тип уравнений по характеристикам. Матрицу квадратичной формы:

$$
A=\left(\begin{array}{ll}
a_{11} & a_{12} \\
a_{12} & a_{22}
\end{array}\right)
$$

при этом, очень удобно использовать для составления уравнения характеристик:

$$
a_{11}(x, y) d y_{2}-2 a_{12}(x, y) d y d x+a_{22}(x, y) d x^{2}=0
$$

Для наглядности покажем на примере дифференциального уравнения в частных производных с постоянными коэффициентами:

$$
\frac{\partial u}{\partial x^{2}}-2 \frac{\partial u}{\partial x \partial y}-3 \frac{\partial u}{\partial y^{2}}+\frac{\partial u}{\partial y}=0
$$

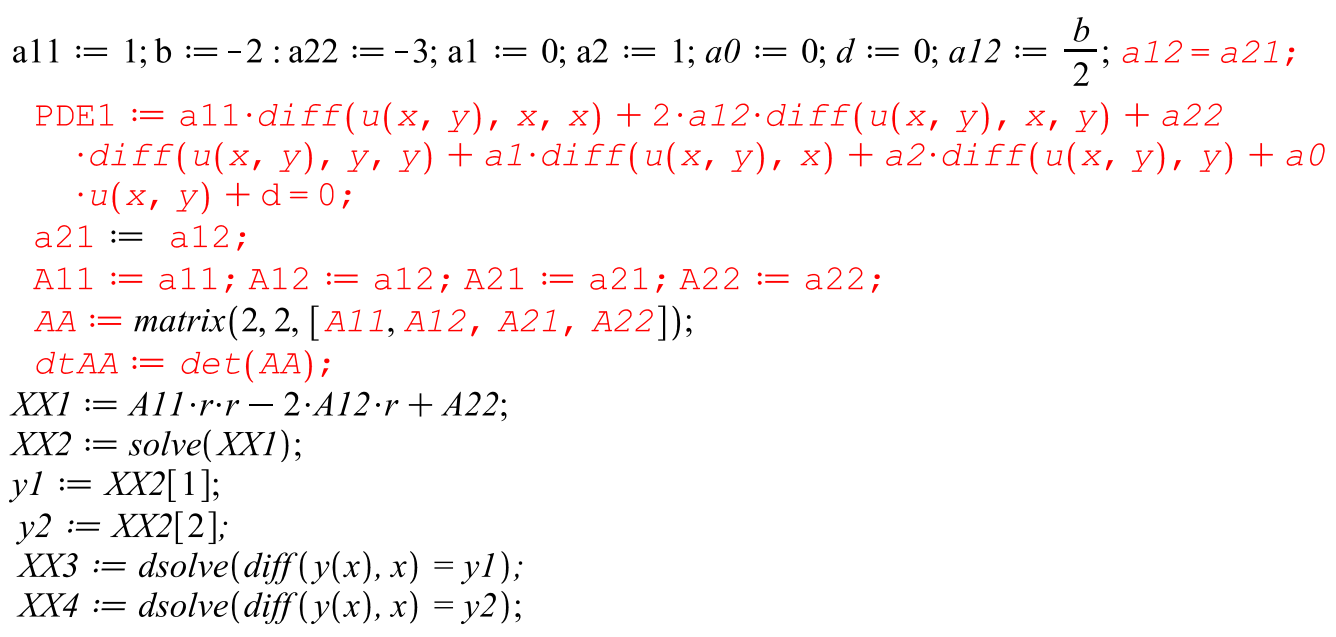

$$
\begin{aligned}
& a 11:=1 \\
& a 22:=-3 \\
& a 1:=0 \\
& a 2:=1 \\
& a 0:=0 \\
& d:=0 \\
& a 12:=-1 \\
&-11=a 21 \\
& P D E 1:=\frac{\partial^{2}}{\partial x^{2}} u(x, y)-2\left(\frac{\partial^{2}}{\partial y \partial x} u(x, y)\right)-3\left(\frac{\partial^{2}}{\partial y^{2}} u(x, y)\right)+\frac{\partial}{\partial y} u(x, y)=0 \\
& a 21:=-1 \\
& A 11:=1 \\
& A 12:=-1 \\
& A 21:=-1
\end{aligned}
$$




\begin{tabular}{l|lrl|l|ll} 
& ISRA (India) & $=\mathbf{1 . 3 4 4}$ & SIS (USA) & $=\mathbf{0 . 9 1 2}$ & ICV (Poland) & $=\mathbf{6 . 6 3 0}$ \\
Impact Factor: & ISI (Dubai, UAE) $=\mathbf{0 . 8 2 9}$ & PUHU (Russia) $=\mathbf{0 . 2 3 4}$ & PIF (India) & $=\mathbf{1 . 9 4 0}$ \\
& GIF (Australia) & $\mathbf{0 . 5 6 4}$ & ESJI (KZ) & $=\mathbf{1 . 0 4 2}$ & IBI (India) & $\mathbf{4 . 2 6 0}$
\end{tabular}

$$
\begin{gathered}
A 22:=-3 \\
A A:=\left[\begin{array}{rr}
1 & -1 \\
-1 & -3
\end{array}\right] \\
d t A A:=-4 \\
X X 1:=r^{2}+2 r-3 \\
X X 2:=1,-3 \\
y 1:=1 \\
y 2:=-3 \\
X X 3:=y(x)=x+\ldots C 1 \\
X X 4:=y(x)=-3 x+\ldots 1
\end{gathered}
$$

Как видно, элементы матрицы используются для уравнения характеристик не определяя тип уравнения. Только значение детерминанта указывает для сведущего, что данное уравнение относится к гиперболическому типу.

Рассмотрим теперь этот же алгоритм на примере дифференциального уравнения в частных производных с переменными коэффициентами:

$$
x \frac{\partial u}{\partial x^{2}}+2(x+y) \frac{\partial u}{\partial x \partial y}+y \frac{\partial u}{\partial y^{2}}=0
$$

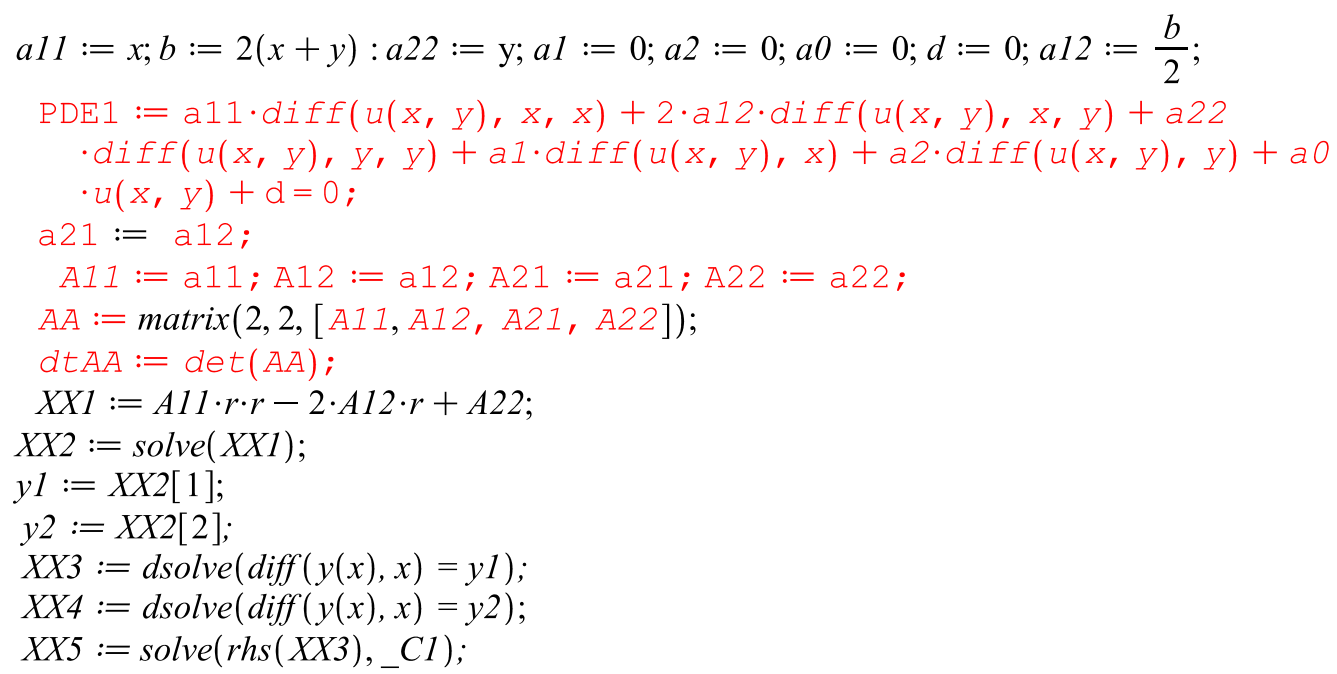

$$
\begin{aligned}
& a 11:=x \\
& a 22:=y \\
& a 1:=0 \\
& a 2:=0 \\
& a 0:=0 \\
& d:=0 \\
& a 12:=x+y \\
& P D E 1:=x\left(\frac{\partial^{2}}{\partial x^{2}} u(x, y)\right)+2(x+y)\left(\frac{\partial^{2}}{\partial y \partial x} u(x, y)\right)+y\left(\frac{\partial^{2}}{\partial y^{2}} u(x, y)\right)=0 \\
& a 21:=x+y \\
& A 11:=x \\
& A 12:=x+y \\
& A 21:=x+y \\
& A 22:=y
\end{aligned}
$$




\begin{tabular}{l|lrl|l|ll} 
& ISRA (India) & $=\mathbf{1 . 3 4 4}$ & SIS (USA) & $=\mathbf{0 . 9 1 2}$ & ICV (Poland) & $=\mathbf{6 . 6 3 0}$ \\
Impact Factor: & ISI (Dubai, UAE) $=\mathbf{0 . 8 2 9}$ & PUHL (Russia) $=\mathbf{0 . 2 3 4}$ & PIF (India) & $=\mathbf{1 . 9 4 0}$ \\
& GIF (Australia) & $\mathbf{0 . 5 6 4}$ & ESJI (KZ) & $=\mathbf{1 . 0 4 2}$ & IBI (India) & $=\mathbf{4 . 2 6 0}$ \\
& JIF & $\mathbf{1 . 5 0 0}$ & SJIF (Morocco) & $=\mathbf{2 . 0 3 1}$ & & \\
\hline
\end{tabular}

$$
\begin{gathered}
A A:=\left[\begin{array}{cc}
x & x+y \\
x+y & y
\end{array}\right] \\
d t A A:=-x^{2}-x y-y^{2} \\
X X 1:=x r^{2}-2(x+y) r+y \\
X X 2:=\left\{r=r, x=\frac{y(2 r-1)}{r(r-2)}, y=y\right\} \\
y 1:=r=r \\
y 2:=x=\frac{y(2 r-1)}{r(r-2)}
\end{gathered}
$$

Определитель квадратичной формы представляет выражение, зависящее как от $x$, так и от $у$ и которое требуется решить для определения типа исходного уравнения, не дойдя до самих характеристик. Следовательно, вызывает определенную трудность решение определителя квадратичной формы исходного уравнения, что и усложняет задачу приведения к каноническому виду дифференциального уравнения в частных прозводных с переменными коэффициентами.

\section{References:}

1. Bitsadze AV (1982) Uravneniya matematicheskoy fiziki. Moscow: Nauka, 1982, $-336 p$.

2. Vladimirov VS (1981) Uravneniya matematicheskoy fiziki. Moscow: Nauka, 1981,-512p.

3. Mikhaylov VP (1983) Differentsial'nye uravneniya $\mathrm{s}$ chastnymi proizvodnymi. M.: Nauka, 1983, -424p.

4. Goloskokov DP (2004) Uravneniya matematicheskoy fiziki. Reshenie zadach $\mathrm{v}$ sisteme Maple uchebnik dlya vuzov - SPb.: Piter, 2004. -539p.

5. D'yakonov VP (2006) Maple 9.5/10 v matematike, fizike i obrazovanii Izd: Piter, 2006.
6. Savotchenko SE, Kuz'micheva TG (2001) S13 Metody resheniya matematicheskikh zadach $\mathrm{V}$ Maple: Uchebnoe posobie - Belgorod: Izd. Belaudit, 2001. - 116 p.

7. Kanatnikov AN, Krishchenko AP, Chetverikov VN (2000) Differentsial'noe ischislenie funktsiy mnogikh peremennykh.M.: Izd-vo MGTU im. N.E. Baumana, 2000.- 456 p.

8. Sdvizhkov OA (2003) - Matematika na komp'yutere: Maple 8 [2003, DjVu, Rus] God vypuska: 2003 Izdatel'stvo: Solon-Press Seriya: Biblioteka studenta ISBN: 5-98003-039-5. 